\title{
MÉTODOS DE MAPPING FEATURES APLICADOS AO PROCESSAMENTO DE SINAIS EEG
}

\section{METHODS OF MAPPING FEATURES APPLIED TO THE PROCESSING OF EEG SIGNS}

\section{André Hallwas Ribeiro Alves; Silvio Antonio Carro; Danillo Roberto Pereira}

\author{
Universidade do Oeste Paulista-UNOESTE, Presidente Prudente, SP \\ Faculdade de Informática de Presidente Prudente - FIPP \\ E-mail: andrehallwas@hotmail.com, \{silvio, danilopereira\}@unoeste.br
}

RESUMO - O eletroencefalograma (EEG) é um exame médico que visa registrar a atividade cerebral do indivíduo para análise posterior. Diversas aplicações estão surgindo atualmente para o mesmo, e um fator de grande importância para qualquer aplicação é encontrar padrões e grupos nos sinais e relacioná-los às ações. Atualmente, existem vários classificadores usados para isso, e esses classificadores são aplicados diretamente aos sinais do EEG. No entanto, outra temática utiliza métodos de Mapping Features no processamento dos sinais e posteriormente, realiza a classificação nos sinais resultantes visando obter resultados melhores.

Palavras-chave: Aprendizado de Máquina; Mapping Features; Inteligência Artificial.

ABSTRACT - The electroencephalogram (EEG) is a medical examination that aims to record the individual's brain activity for further analysis. Several applications are currently emerging for the same, and a major factor for any application is finding patterns and groups in the signals and relating them to the actions. Currently, there are several classifiers used for this, and these classifiers are applied directly to EEG signals. However, another theme uses Mapping Features methods in signal processing and then performs the classification on the resulting signals for better results.

Keywords: Machine Learning; Mapping Features; Artificial Intelligence. 


\section{INTRODUÇÃO}

O eletroencefalograma (EEG) é um exame médico que permite o estudo e o registro das correntes elétricas desenvolvidas no cérebro durante diversas atividades exercidas pelo indivíduo. Começou a ser realizado após a descoberta de Hans Berger (BERGER, 1929) (COLLURA, 1993) de que o cérebro gera uma atividade elétrica capaz de ser registrada como visto na Figura 1. 0 exame é feito através de um dispositivo que amplifica os sinais elétricos milhares de vezes e estes são registrados e armazenados em uma base de dados, para posteriormente serem analisados.

Figura 1. Sinais de EEG

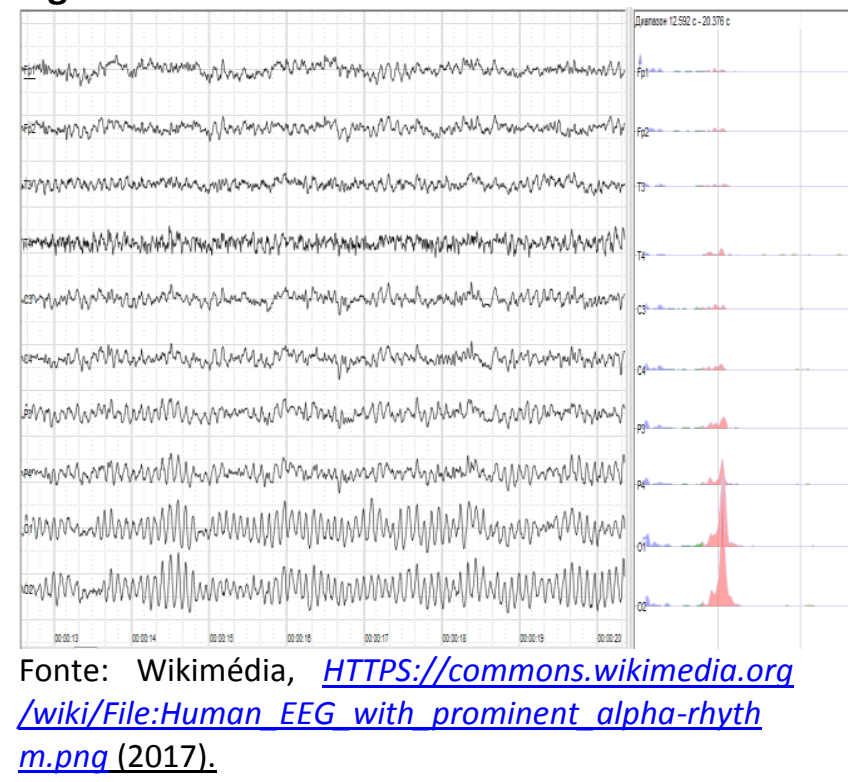

As aplicações mais comuns para EEG são no diagnóstico de epilepsia e na identificação de derrames ou tumores no cérebro. Porém o EEG possui uma ampla gama de aplicações, e atualmente estão surgindo novas aplicações relacionadas à identificação de padrões nos sinais obtidos pelo EEG como na Biometria (DELPOZOBANOS, TRAVIESO, et al., 2015), no reconhecimento de estado mental (BASHIVAN, RISH e HEISIG, 2016), no aprimoramento de atenção (CHO, JANG, et al., 2002), na psicofisiologia (BAUMGARTNER, VALKO, et al., 2006), na navegação em ambientes de realidade virtual (RV) ( $F$.
BISCHOF e BOULANGER, 2003) (ZHAO, ZHANG e CICHOCKI, 2009) (LEEB, FRIEDMAN, et al., 2004) (LEEB, FRIEDMAN, et al., 2007) (LIN, CHUNG, et al., 2007 JULY), e no controle e para a classificação de indivíduos baseando-se na atividade neural (YEOM, SUK e LEE, 2013), dentre outras.

No entanto um fator de grande importância para qualquer uma das aplicações é encontrar padrões nos sinais EEG. Afinal, para qualquer que seja a aplicação, esta necessita de uma profunda análise nos sinais de EEG a fim de descobrir um padrão (grupo nos sinais do EEG) que represente algo de interesse. Entre alguns exemplos está o diagnóstico de epilepsia que atualmente é a aplicação mais comum do exame de EEG. Este diagnóstico consiste em encontrar um grupo de sinais do EEG que é comum a indivíduos com epilepsia no exame de EEG da pessoa. Outro exemplo disto é na navegação em ambientes de RV onde a análise dos sinais de EEG visa encontrar padrões que representem a movimentação de um indivíduo para posteriormente transportar estes movimentos para a aplicação. E também na biometria para a classificação de indivíduos baseando-se nas atividades neurais (YEOM, SUK e LEE, 2013). Onde a análise nos sinais de EEG busca discriminar indivíduos de outros indivíduos com base em sua atividade neural captada pelo exame de EEG. Portanto, é de extrema importância encontrar métodos que auxiliem na busca por padrões nos sinais do EEG.

$E$ atualmente existem diversos classificadores que são utilizados para a identificação e discriminação de padrões e grupos nos sinais de EEG como os descritos em (DELPOZO-BANOS, TRAVIESO, et al., 2015). E estes classificadores são aplicados nos sinais EEG durante a classificação dos sinais, conforme observado na Figura 2. No entanto, eles também podem ser aplicados em conjunto com diversos métodos de separação e agrupamento de dados, e alguns destes métodos são os de Mapping Features (Ferramentas de Mapeamento de Atributos). Sendo assim em uma outra temática, os 
métodos de Mapping Features são utilizados no processamento dos sinais de EEG, e posteriormente são aplicados os classificadores nos sinais resultantes, como visto na Figura 2.

Figura 2. Métodos de busca de padrões nos sinais de EEG

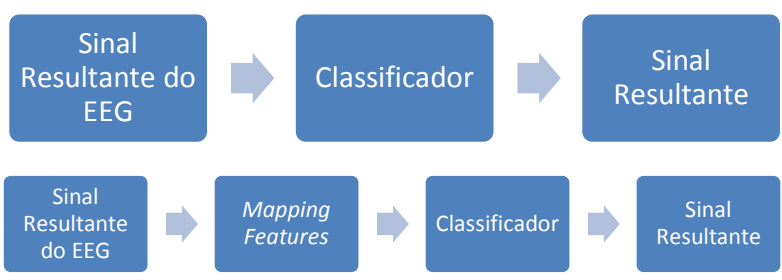

Fonte: Próprio autor.

Métodos de Mapping Features possuem diversas aplicações, mas poucas têm como principal foco os sinais de EEG. No entanto, eles são um recurso muito útil para auxiliar na separação e agrupamento de dados nos sinais de EEG. Pois os métodos de Mapping Features têm a capacidade de melhorar a precisão dos resultados obtidos pelos classificadores na identificação e discriminação de padrões, trazendo assim melhores resultados.

Os métodos de Mapping Features utilizados no contexto deste trabalho são: (i) CDT (Cumulative Distribution Transform), (ii) LPC (Linear Predictive Codins), e (iii) MFCC (Mel-Frequency Cepstral Coefficients). O CDT (PARK, KOLOURI, et al., 2015) (KOLOURI, PARK e K. ROHDE, 2015) é um espaço utilizado para diferenciar as classes de dados representadas nele. Consegue realizar a separação de certos tipos de problemas de classificação linearmente no espaço (por exemplo, problemas de Fourier e Wavelet (PARK, KOLOURI, et al., 2015) (KOLOURI, PARK e K. ROHDE, 2015)), e possui uma baixa expectativa de erro. O LPC (LUIZ A. CARVALHO e DIAS, 2000) e (RABINER, JUANG e $B, 1993)$ é um dos principais codificadores de áudio, e é uma compressão com perda de qualidade (lossy) de áudio. Ele consegue diminuir significativamente a taxa de transmissão de um áudio, sem alterar as características perceptíveis. O MFCC (R. FACHINI e ROBERTO HEINEN, 2016)
(LINDASALWA, BEGAM KASIM RAWTHAR e L., 2010) (D. R. CUADROS, CATALDO, et al., 2007) (BRENT, 2010) (TZANETAKIS e COOK, 2002) é uma ferramenta extremamente útil para diferenciar ou encontrar semelhanças entre grupos de objetos. Geralmente é utilizado para reconhecimento de voz e converte um grande sinal com alta amostragem em coeficientes que demonstram características do sinal em determinado intervalo.

Sendo assim foram aplicados os métodos de Mapping Features no processamento dos sinais de EEG visando obter melhores resultados na tarefa de classificação.

\section{MATERIAIS E MÉTODOS}

Esta seção descreve as ferramentas, métodos e objetos utilizados na pesquisa.

\subsection{LINEAR PREDICTIVE CODINS}

O LPC (Codificação Por Predição Linear) é um codificador paramétrico para sinais de voz humana. E uma das técnicas para análise de fala e processamento de sinais de áudio mais poderosas atualmente. É um dos métodos mais úteis para codificar sinais de áudio de uma boa qualidade a uma baixa taxa de bits. Fornece estimativas precisas de parâmetros de áudio, é utilizado principalmente para processamento de sinais de áudio. Porém também é utilizado em aplicações de diversas áreas, entre estas vale destacar a identificação de abalos sísmicos, identificação de desgaste de ferramentas (TEIXEIRA DOS SANTOS, ROSETTI DE ALMEIDA e CHIEN CHING TU, 1999), processamento de sinais (TEIXEIRA DOS SANTOS, ROSETTI DE ALMEIDA, et al., 1997), reconhecimento de voz (FACHETTI BONGIOVANI e SALOMAO, 2017), e o processamento de eletrocardiogramas (ECG) (MACHADO, 1995). O LPC é uma técnica de compressão de audio lossy. Porém, consegue obter uma grande redução na taxa de transmissão, teoricamente sem perda de qualidade perceptível ao ouvido humano. 
Ele gera um filtro de síntese diferente para cada bloco do sinal de 10 a 30 Milissegundos ( $\mathrm{ms}$ ) de duração. $\mathrm{E}$ ao invés de armazenar informações de cada amostra (Sample) do sinal de áudio, são armazenados apenas alguns coeficientes. Desta forma o LPC faz uma compressão, diminuindo o sinal, e mantendo as principais características perceptíveis.

O LPC analisa a forma da onda para produzir um filtro de síntese que varia no tempo, e modela o trato vocal reproduzindo sua função de transferência ilustrada na Figura 3. A análise do LPC consiste na obtenção de coeficientes preditores produzidos através do filtro correlator que processa segmentos curtos do sinal, de 10 a $30 \mathrm{~ms}$ por padrão. Desta forma a análise se baseia geração de um modelo linear para o mecanismo de produção do sinal. Assim a Excitação (o Sinal) $U(n)$ é a entrada do sistema, e $S(n)$ é a fala sintetizada ou a saída, e basicamente $U(n)$ passa pelo modelo do trato vocal produzido $H(z)$ e produz $S(n)$ que é o sinal resultante.

Figura 3. Modelo de produção de Som.

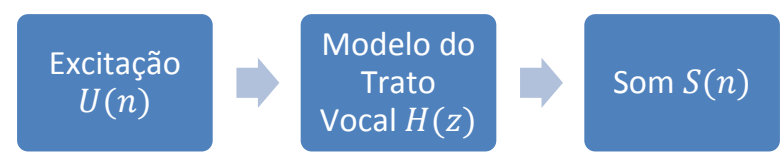

Fonte: Próprio autor.

O Modelo do Trato vocal $H(z)$ é obtido por um filtro digital linear de polos e variante no tempo como visto na Equação 1.

$$
H(z)=\frac{1}{A(z)}
$$

Onde, $A(z)$ é dado pela Equação 2:

$$
A(z)=1-\sum_{i=1}^{\rho} \alpha_{i} z^{-i}
$$

Os parâmetros $\alpha_{i}$ são os coeficientes preditores $\left(\alpha_{i}=\left[a_{1}, a_{2} \ldots a_{p}\right]^{t}\right)$. O filtro de síntese $H(z)$ tem a função de inserir no sinal sintetizado a correlação a curto termo encontrada no sinal original. Que é a análise short-term Prediction (STP) na qual o cálculo dos coeficientes preditores pode ser realizado da forma apresentada a seguir (LUIZ A. CARVALHO e DIAS, 2000).

Segundo (LUIZ A. CARVALHO e DIAS, 2000) e (RABINER, JUANG e B, 1993) Inicialmente o preditor realiza uma soma ponderada ou a média das últimas $P$ amostras visando estimar a amostra presente, como visto na Equação 3 pode-se reescrever a amostra $S(n)$ como:

$$
S_{(n)}=\sum_{i=1}^{P} \alpha_{i} S_{n-i}+e_{n}
$$$$
\text { Onde } S_{n-i} \text { são amostras }
$$

anteriores, $S_{(n)}$ é a amostra atual, e $e_{n}$ é o erro de predição. Os coeficientes são calculados de modo a minimizar a energia média $E$ do sinal $e_{n}$ como visto na Equação 4:

$$
\begin{gathered}
E=\sum_{\substack{n=1 \\
\text { Onde, } \alpha_{0}}}^{N} e_{n}^{2}=\sum_{n=1}^{N}\left(\sum_{i=0}^{P} \alpha_{i} s_{n-i}\right)^{2} \text { é o número de }
\end{gathered}
$$
amostras contidas na janela de tempo pela qual o erro de predição é computado. Os valores padrões são uma janela de 10 a 30 ms correspondendo a um valor de $N=80$ amostras. E a respeito de um coeficiente $\alpha_{m}$, se faz necessário fazer sua derivada parcial igual a zero como é mostrado nas Equações 5 e 6:

$$
\begin{gathered}
\frac{\partial E}{\partial \alpha_{m}}=\sum_{n-1}^{N} 2 s_{n-m} \sum_{i=0}^{P} \alpha_{i} s_{n-i}=0 \\
=\sum_{i=0}^{P} \sum_{n=1}^{N} s_{n-m} s_{n-i} \alpha_{i}=0 \\
\text { Onde } m=1,2,3, \ldots, 10 .
\end{gathered}
$$

A soma mais interna pode ser interpretada como o coeficiente de correlação $C_{i m}$, e reescrevendo obtêm-se a Equação 7:

$$
\sum_{i=1}^{P} C_{i m} \alpha_{i}=C_{0 m}
$$

Após determinar os coeficientes de correlação $C_{i m}$, a Equação 7 pode ser usada para determinar matricialmente os coeficientes preditores, pois se trata de um sistema linear de equações e variáveis. Um sintetizador realiza a tarefa de recriar o sinal através passagem de uma excitação 
especifica pelo mesmo modelo matemático do trato vocal que é gerado periodicamente.

Os coeficientes preditores são atualizados aproximadamente a cada 20 $30 \mathrm{~ms}$, e são enviados em conjunto com os demais parâmetros do sistema o flag de segmento sonoro, o período de pitch, no caso de segmento do sinal, o fator de ganho de excitação G, determinará a amplitude do sinal de saída gerado pelo filtro de síntese. Após serem codificados de forma binária, os coeficientes do filtro de síntese e os parâmetros descritos acima são gerados a uma taxa de relativamente baixa, e o resultado é um sinal de qualidade razoável preservando as principais características do sinal original, levando-se em conta a relativamente baixa taxa de bits.

\subsection{MEL-FREQUENCY \\ CEPSTRAL COEFFICIENTS}

$O$ MFCC é uma técnica utilizada para o processamento de sinais é geralmente utilizado para tarefas de reconhecimento de voz, fala e sons (R. FACHINI e ROBERTO HEINEN, 2016) (LINDASALWA, BEGAM KASIM RAWTHAR e L., 2010) (D. R. CUADROS, CATALDO, et al., 2007), aplicações musicais (TZANETAKIS e COOK, 2002) (LOGAN), e processamento de imagens (GUPTA, JAAFAR, et al., 2013). O MFCC considera a percepção não linear do som pelo ouvido humano, e o que torna o uso do MFCC interessante é o fato de sua aplicação reduzir um espectro de pontos e estes pontos podem ser utilizados para verificar a similaridade ou a distinção de sons (BRENT, 2010).

Para o realizar o processamento de um sinal através do MFCC como visto na Figura 4 é inicialmente realizado o processo de pré-ênfases onde o sinal é preparado e filtrado, após isto é realizado a segmentação, o janelamento e aplicado a Transformada Rápida de Fourier (FFT). A amplitude da FFT é filtrada por janelas triangulares na escala Mel e então aplica-se o logaritmo. A Transformada Discreta do Cosseno (TDC) é aplicada no resultado, e os Coeficientes MelCepstrais são as amplitudes resultantes dos processos realizados (LOGAN) (R. FACHINI e ROBERTO HEINEN, 2016).

Figura 4. Diagrama de Fases do MFCC

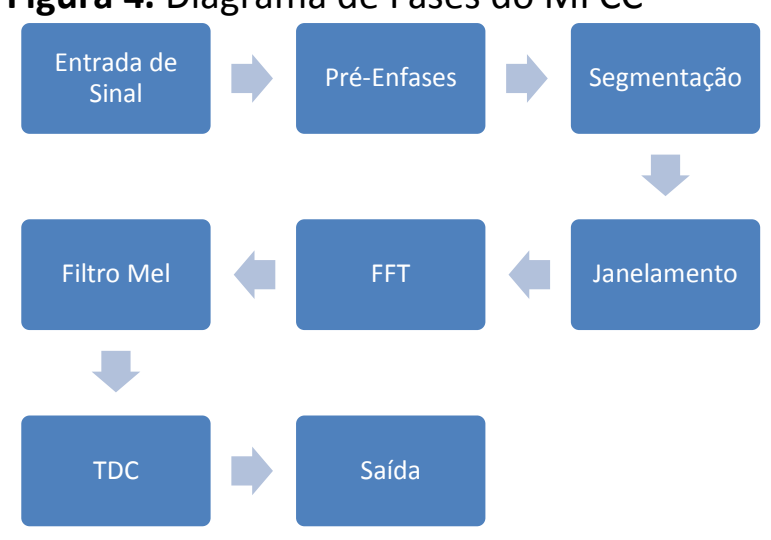

Fonte: Próprio autor.

O Primeiro passo consiste em realizar a computação dos coeficientes para processar a passagem do sinal de entrada através de um filtro que enfatiza as frequências mais altas. Este processo irá aumentar a energia do sinal de maior frequência, Como: $\quad Y_{(n)}=X_{(n)}-$ $0.95 X(n-1)$ (LINDASALWA, BEGAM KASIM RAWTHAR e L., 2010) (PAL SINGH e RANI, 2014) (W., C., et al., 2006), é preciso fazer a segmentação do sinal continuo em pequenos quadros de $N$ amostras geralmente $N=100$ e $M=256(N<M)$ sendo $M$ os quadros e $N$ as amostras (GUPTA, JAAFAR, et al., 2013), a seguir é necessário atenuar as descontinuidades e interrupções no início e no final de cada segmento, e para isto multiplica-se os quadros por uma função de janelamento $W(n)$. Existem diversas funções de janelas, porém uma das principais funções utilizadas para aplicações de processamento de sinais de áudio é a janela de Hamming, que geralmente é definida como visto na Equação 8:

$$
\begin{gathered}
W(n)=0.54-0.46 \cos \frac{2 \pi n}{N-1} \\
0 \leq n \leq N-1
\end{gathered}
$$

Onde a janela é definida como $W(n), 0 \leq n \leq N-1, N$ representa a quantidade de amostras em cada quadro, o sinal de saída após o janelamento é representado como $Y(n)=X(n) *$ $W(n), 0 \leq n \leq N-1, \quad$ e $\quad Y(n)$ 
representa o sinal de saída depois da multiplicação do sinal de entrada representado como $X(n)$, e a janela de Hamming é representada como $W(n)$.

Após o janelamento do sinal a FFT é utilizada para fazer a conversão de cada quadro contendo $N$ amostras do domínio de tempo para o domínio de frequência. A FFT recebe como entrada um sinal de tamanho $N$ produzindo como saída um sinal de mesmo tamanho representando os coeficientes espectrais, ou espectro de Magnitude. A Transformada Rápida de Fourier é um algoritmo rápido para aplicar a Transformada Discreta de Fourier (DFT), sobre o conjunto dado de $N$ amostras mostrado na Equação 9:

$$
D_{k}=\sum_{n=0}^{N_{n-1}} D_{n} e^{\frac{-j 2 \pi k n}{N_{n}}}
$$

$$
\text { Se } k=0,1,2 \ldots N_{n-1}
$$

Assim, nesta equação $D_{n}$ é o sinal de entrada, e $\frac{-j 2 \pi k n}{N_{n}}$ a função base que define os valores complexos para cada ponto $D_{k}$ no domínio de frequência.

A escala Mel é uma escala psicoacústica (ZWICKER e FASTL, 1999) que explora a relação de percepção da frequência fundamental entre dois tons. Criada a partir do estudo da dinâmica do sistema auditivo humano. A unidade de medida Mel (em referência a melodia) refere-se à frequência subjetiva de tons puros percebida pelo ouvido humano (BRENT, 2010) (R. FACHINI e ROBERTO HEINEN, 2016). O mapeamento entre $f$ e a frequência percebida Mel pode ser dado de diversas formas neste caso é dado pela Equação 10:

$$
\operatorname{Mel}(f)=1,127 * \ln \left(1+\frac{f}{700}\right)
$$

Onde $\mathrm{Mel}(f)$ são os coeficientes mel.

Para criar o banco de frequências $\mathrm{Mel}$, utiliza-se de filtros passa-banda com envelopes triangulares, com o centro dos filtros espaçados de acordo com a escala Mel. Assim enfatizando as frequências próximas ao centro do filtro (onde ocorre a mudança perceptiva de tom) e atenuando as frequências vizinhas (R. FACHINI e ROBERTO HEINEN, 2016). A Figura 5 mostra um banco de filtros típico para calcular o espectro de frequências mel.

Figura 5. Banco de Filtros com 25 filtros de passa-banda triangulares para calcular 0 espectro de frequências de mel.

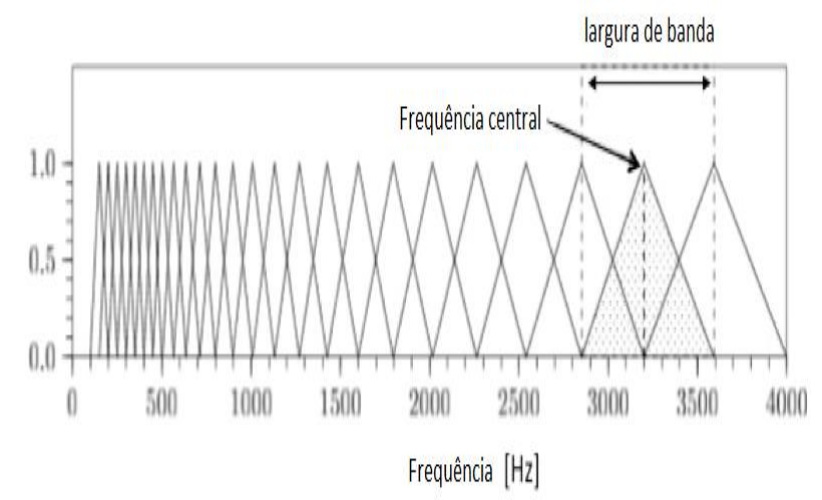

Fonte: Modificado de http://recognize-speech.com/ feature-extraction/mfcc (2017).

O último passo para a obtenção dos Coeficientes Mel-Cepstrais tem por objetivo converter o espectro mel de volta para o domínio espacial. Para isto é aplicada a DCT na Equação 11 aos Componentes gerados pela aplicação do banco de filtros. A saída após a aplicação da DCT é conhecida como MFCC.

$$
C_{n}=\sum_{k-1}^{k}\left(\log D_{k}\right) \cos \left[m\left(k-\frac{1}{2}\right) \frac{\pi}{k}\right]
$$

Onde $m=0,1 \ldots k-1, C_{n}$ representa o MFCC e $m$ é o número de coeficientes, assim o número total de coeficientes extraídos a partir de cada quadro é de $m$ coeficientes (BRENT, 2010) (R. FACHINI e ROBERTO HEINEN, 2016).

\subsection{CUMULATIVE \\ DISTRIBUTION}

\section{TRANSFORM}

O CDT (PARK, KOLOURI, et al., 2015) (KOLOURI, PARK e K. ROHDE, 2015) é uma variação da Função de distribuição cumulada cuja função é transformar um sinal linear não bijectivo a partir do espaço de densidades para um espaço de funções diferenciáveis. A Função de distribuição acumulada (FDA) é um método de probabilidade que nos dá uma forma de descrever como as probabilidades são relacionadas aos valores ou aos intervalos de valores de variáveis aleatórias. 
De forma geral a FDA calcula a probabilidade acumulada de um determinado valor de $\mathrm{x}$ em um espaço. Desta forma é possível utilizar a FDA para determinar a probabilidade de que uma determinada observação aleatória extraída de um sinal seja menor ou maior que um determinado valor ou pertença a um intervalo de valores, a FDA é descrita na Equação 12 e 13.

$$
I \leq X \leq N, X \leq N, \text { ou } X \geq N
$$

Onde $I$ representa o valor inicial do intervalo, $\mathrm{N}$ é o valor máximo do intervalo, e $\mathrm{X}$ é a probabilidade resultante da FDA.

A FDA é denotada por:

$$
F_{(x)}=P(X \leq x) x \in R .
$$

Onde $F(x)$ é a probabilidade de que a variável $X$ assume um valor menor que $\mathrm{x}$, onde para cada $x$ a função $F$ assumira um valor diferente.

A função de distribuição é obtida a partir da função de probabilidade associada, no caso de uma variável aleatória é vista na Equação 14:

$$
f(x)=\int_{-i n f}^{x} f(x i) d x
$$

Onde $F(x)$ segue as mesmas propriedades de (14). Sendo que $x i$ são os valores no intervalo da integral. Assim considerando duas funções de probabilidade de densidade $f_{1}$ e $f_{2}$ e considerando que $f_{1}$ é uma referência de densidade, então é possível relacionar $f_{2}$ para obter a densidade de $f_{x}$, e utilizando a Equação 14 é possível definir o CDT de $f_{2}$ como visto na Equação 15.

$$
f_{3}(x)=\left(f_{2}(x)-x\right) \sqrt{f_{1}(x)}
$$

Sendo que $f_{1}(x)$ pode ser reescrito como na Equação 16 e $f_{1}(x)$ é descrito na Equação 17.

$$
\begin{gathered}
\theta\left(f_{1}(x)\right)=x \\
f_{1}(x)=\theta^{-1}(x)
\end{gathered}
$$

Sendo assim ao substituir a Equação 16 na Equação 15, é possível encontrar o CDT de $f_{1}(x): X \rightarrow R$ (PARK, KOLOURI, et al., 2015) (KOLOURI, PARK e K. ROHDE, 2015).

\subsection{ANÁLISE E PREPARAÇÃO DO AMBIENTE}

Antes da aplicação da metodologia foi preparado o ambiente adequado para o desenvolvimento da pesquisa. Para isto foi realizada uma pesquisa sobre as ferramentas de Mapping Features visando realizar a implementação na linguagem de programação Python ou encontrar frameworks, e sobre a base de dados que seria utilizada como entrada para os métodos que foram utilizados. Inicialmente foi realizado um levantamento de trabalhos na área visando analisar os resultados antes da aplicação dos métodos de mapeamento. Em seguida, foram realizados testes prévios sem o uso da metodologia visando verificar os resultados obtidos na base de dados utilizada.

\subsection{BASE DE DADOS}

A base de dados (SCHALK, MCFARLAND, et al., 2004) (GOLDBERGER, AMARAL, et al., 2000) contém amostras de 6 atividades praticadas por 109 pessoas. Cada amostra de atividade possui 64 canais como visto na Figura 6, com 80 amostras de áudio por segundo. O Tempo de cada amostra de atividade varia entre 1 e 2 minutos.

As duas primeiras atividades correspondem a pessoas com os olhos abertos e fechados. A terceira, sétima e décima primeira atividade corresponde a pessoas abrindo e fechando o punho esquerdo ou o direito. A quarta, oitava e décima segunda atividade corresponde a pessoas imaginando abrir e fechar o punho esquerdo ou o direito. A quinta, nona e décima terceira atividade corresponde a pessoas abrindo e fechando os punhos ou os dois pés. A sexta, décima e décima quarta atividade corresponde a pessoas imaginando abrir e fechar os punhos ou os dois pés. 
Figura 6. Canais capturados pelo exame de EEG durante as atividades realizadas.

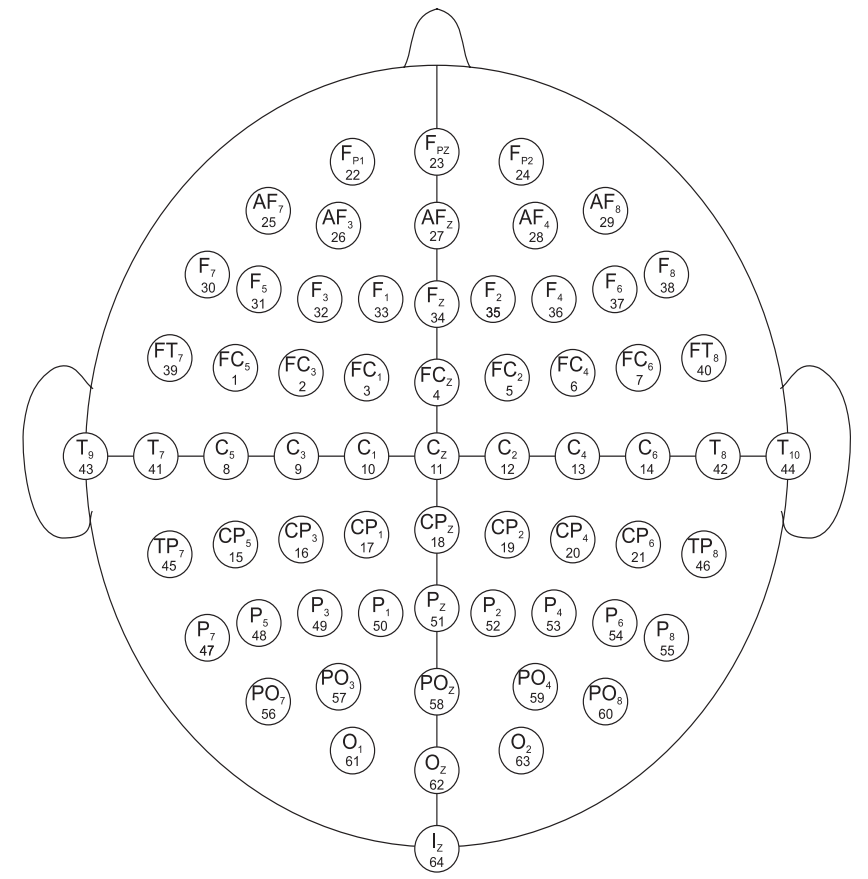

Fonte: Obtido de https://www.physionet.org/pn4/eeg mmidb/64 channel sharbrough.png (2018).

\subsection{CLASSIFICADORES}

Foram utilizados classificadores como Support Vector Machine (SVM) (HSU e CHANG, 2003), K-nearest neighbors (KNN) (COVER e HART, 1967), Naive Bayes (MCCALLUM e NIGAM, 1998), e Random Forest (DENISKO D, 2018) para a tarefa de classificação dos sinais após o processamento.

\subsection{APLICAÇÃO DAS FERRAMENTAS DE MAPPING FEATURES ISOLADAMENTE}

Foram aplicadas as ferramentas de Mapping Features LPC, MFCC e CDT separadamente de forma a buscar o melhor resultado individualmente como observado na Figura 7.

Desta forma, no primeiro método de aplicação das ferramentas, o codificador LPC foi aplicado nos sinais resultantes do EEG. Em sua aplicação comum o LPC é utilizado no tratamento de áudio e gera um modelo do trato vocal, assim sendo capaz de recriar o sinal de áudio inicial com suas principais características. No entanto aqui é obtido um modelo que representa os sinais de EEG para cada espaço de tempo analisado, e então sinal é recriado através do codificador LPC. Assim fazendo uso da capacidade do LPC de gerar um sinal compacto e que mantem as principais características do sinal original. $\mathrm{E}$ então o sinal resultante foi classificado.

No segundo método foi aplicado o MFCC, outro método originado no processamento digital de áudio. Quando utilizado no sinal de EEG é possível obter os coeficientes mel-Cepstrais que em sua aplicação original são utilizados para o reconhecimento de voz. No entanto neste caso os MFCC são utilizados para obter dados que representam padrões nos sinais obtidos do EEG. Após isto os sinais resultantes são classificados.

E por fim no terceiro método o CDT foi aplicado no sinal de EEG, e o sinal resultante foi classificado.

\subsection{APLICAÇÃO DAS FERRAMENTAS DE MAPPING FEATURES EM CONJUNTO}

As ferramentas de Mapping Features foram aplicadas em conjunto de forma buscar a combinação destas ferramentas que produz o melhor resultado como visto na Figura 7.

No primeiro método realizado em conjunto, o LPC foi aplicado nos sinais de EEG e o CDT no sinal resultante do LPC, o sinal resultante foi classificado. No segundo método o LPC foi aplicado no sinal de EEG e então o MFCC foi aplicado no sinal resultante do LPC, após isto o sinal resultante foi classificado. No terceiro método o MFCC foi aplicado nos sinais de EEG e o CDT foi aplicado nos sinais resultantes, após isto, o sinal resultante foi classificado. No quarto método o MFCC foi aplicado no sinal de EEG da mesma forma que no terceiro método, porém após isto foi aplicado o LPC e assim o sinal resultante foi classificado.

No quinto método foi aplicado o CDT no sinal de EEG, e então no sinal resultante o MFCC foi aplicado, e após isto o sinal resultante foi classificado. No sexto método o CDT foi aplicado no sinal de EEG da mesma forma que o quinto método, no entanto será 
- LPC foi aplicado nos sinais gerados pelo CDT, e então o sinal resultante foi classificado.

Figura 7. Ciclo dos métodos aplicados aos sinais de EEG.

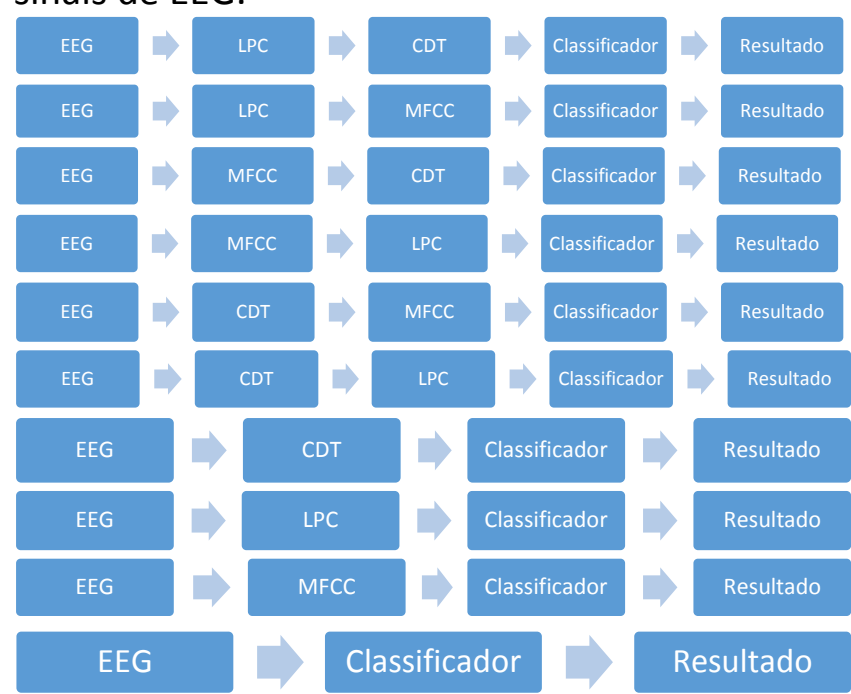

Fonte: Próprio autor.

Por fim no último método foram aplicadas todas as combinações das 3 ferramentas de Mapping Features nos sinais de EEG e após isto a classificação foi realizada nos sinais resultantes. Dessa forma, foram aplicados todos os métodos com o objetivo de verificar a melhor combinação dos mesmos.

\section{RESULTADOS E DISCUSSÃO}

Inicialmente foram feitos testes sem nenhum método de Mapping Features visando estabelecer um resultado base. Metodologias que obtiveram resultados muito inferiores ao resultado base foram descartadas. Apenas foram consideradas metodologias que obtiveram resultados com classes balanceadas.

Nos testes realizados com duas atividades olhos abertos e olhos fechados, sem nenhum método de Mapping Features foi obtido um máximo de $53 \%$ de precisão com o Random Forest. Com o uso do LPC foi obtida uma precisão máxima de $59 \%$ com o classificador SVM. Com o uso do MFCC e o CDT combinados foi obtida uma precisão máxima de $60 \%$ com o Random Forest. Com o uso do MFCC foi obtida uma precisão máxima de $82 \%$ com o classificador Random Forest. Conforme visto na Tabela 1.

Tabela 1. Precisão por Algoritmo. Teste feito em base de dados com 109 pessoas e 2 atividades. Olhos abertos e Olhos Fechados.

\begin{tabular}{|l|l|l|l|l|}
\hline Classificador & Base & LPC & $\begin{array}{l}\text { MFCC E } \\
\text { CDT }\end{array}$ & MFCC \\
\hline $\begin{array}{l}\text { Random } \\
\text { Forest }\end{array}$ & $53 \%$ & $56 \%$ & $60 \%$ & $82 \%$ \\
\hline Naive Bayes & $50 \%$ & $54 \%$ & $58 \%$ & $82 \%$ \\
\hline KNN & $50 \%$ & $55 \%$ & $57 \%$ & $81 \%$ \\
\hline SVM & $50 \%$ & $59 \%$ & $58 \%$ & $80 \%$ \\
\hline
\end{tabular}

Fonte: Próprio Autor.

Nos testes realizados com todas as atividades da base de dados sem nenhum método de Mapping Features foi obtida uma precisão máxima de $25 \%$ com o classificador SVM. Com o uso do LPC foi obtida uma precisão média de $21 \%$. Com o uso do MFCC e do CDT combinados foi obtida uma precisão máxima de $22 \%$ com o SVM. Nos testes realizados com o MFCC foi obtida uma precisão máxima de $31 \%$ com os classificadores KNN, e o Random Forest. Conforme visto na Tabela 2.

Com o uso de Mapping Features houve uma grande redução no tempo de classificação. Pois estes métodos com exceção do LPC reduzem o tamanho do sinal, evidenciando as melhores características.

Tabela 2. Precisão por Algoritmo. Teste feito em base de dados com 109 pessoas e 6 atividades.

\begin{tabular}{|l|l|l|l|l|}
\hline Classificador & Base & LPC & $\begin{array}{l}\text { MFCC E } \\
\text { CDT }\end{array}$ & MFCC \\
\hline $\begin{array}{l}\text { Random } \\
\text { Forest }\end{array}$ & $23 \%$ & $21 \%$ & $20 \%$ & $31 \%$ \\
\hline Naive Bayes & $23 \%$ & $21 \%$ & $20 \%$ & $29 \%$ \\
\hline KNN & $20 \%$ & $20 \%$ & $21 \%$ & $31 \%$ \\
\hline SVM & $25 \%$ & $21 \%$ & $22 \%$ & $27 \%$ \\
\hline
\end{tabular}

Fonte: Próprio Autor.

Em ambos os casos o MFCC obteve o melhor desempenho. O MFCC consegue extrair de forma mais eficaz as características 
dos sinais de EEG nos casos analisados, devido a sua capacidade de gerar uma assinatura do sinal ao realizar a redução do sinal. Com isso, os sinais podem ser diferenciados com maior precisão. E devido a isto, os classificadores obtêm um melhor resultado.

\section{CONSIDERAÇÕES FINAIS}

O uso desta metodologia produziu resultados melhores nos casos analisados. Isto se deve principalmente ao fato dos métodos de Mapping Features obterem melhores resultados na extração de características dos sinais de EEG. O uso desta metodologia também proporcionou mais eficiência na classificação devido ao fato que estes métodos reduzem o tamanho dos sinais.

\section{REFERÊNCIAS}

BASHIVAN, P.; RISH, I.; HEISIG, S. Mental State Recognition via Wearable EEG. CoRR, 2016. abs/1602.00985.

BAUMGARTNER, T. et al. Neural Correlate of Spatial Presence in an Arousing and Noninteractive Virtual Reality: An EEG and Psychophysiology Study. [S.I.]: CYBERPSYCHOLOGY \& BEHAVIOR 9, Number 1 , 2006.

https://doi.org/10.1089/cpb.2006.9.30

BERGER, H. Ueber das Elektroenkephalogramm des Menschen. Archiv für Psychiatrie und Nervenkrankheiten, v. 87, n. 1, p. 527-570, 1929. https://doi.org/10.1007/BF01797193

BRENT, W. Physical and perceptual aspects of percussive timbre. 2010. Tese(Doutorado). - University of California, 2010.

$\mathrm{CHO}, \mathrm{B} . \mathrm{H}$. et al. Attention Enhancement System using Virtual Reality and EEG
Biofeedback. Virtual Reality Conference, 2002. 10.1109/VR.2002.99651, IEEE. 0. 156.

COLLURA, T. F. History and Evolution of Electroencephalographic Instruments and Techniques. Journal of Clinical Neurophysiology, v. 10 , n. 4, p. 476-477, 1993. https://doi.org/10.1097/00004691199310000-00007

COVER, T. M.; HART, P. E. Nearest neighbor pattern classification. IEEE Transactions on Information Theory. v.13, n. 1 p. 21-27., 1967.

https://doi.org/10.1109/TIT.1967.1053964

CUADROS, D. R. C. et al. Comparação entre as técnicas de MFCC e ZCPA para reconhecimento robusto de locutor em ambientes ruidosos. In: SBMAC - SOCIEDADE BRASILEIRA DE MATEMÁTICA APLICADA E COMPUTACIONAI 30., 2007, Florianopolis. Anais [...]. Florianópolis, SC, 2007.

DELPOZO-BANOS, $M$. et al. EEG biometric identification: a thorough exploration of the time-frequency domain. Journal of Neural Engineering, v. 12, n. 5 id. 056019, 2015. https://doi.org/10.1088/17412560/12/5/056019

DENISKO D, H. M. Classification and interaction in random forests. Proceedings of the National Academy of Sciences of the United States of America. v. 115, n. 8, p. 1690-1692, 2018. doi:10.1073/pnas.1800256115., 2018. https://doi.org/10.1073/pnas.1800256115

F. BISCHOF, W.; BOULANGER, P. Spatial Navigation in Virtual Reality Environments: An EEG Analysis. Cyberpsychology \& Behavior, v. 6, n. 5, 2003. https://doi.org/10.1089/1094931037697105 $\underline{14}$

FACHETTI BONGIOVANI, G.; SALOMAO, J. Reconhecimento de comandos de voz no acionamento de eletrodomésticos e equipamentos domicilares. Vitória: Instituto 
Federal de Educação Ciência e Tecnologia do Espírito Santo, 2017.

GOLDBERGER, A. et al. PhysioBank, PhysioToolkit, and PhysioNet: Components of a New Research Resource for Complex Physiologic Signal. Circulation v. 101, n. 23, p. e215-e220

http://circ.ahajournals.org/cgi/content/full/1 01/23/e215], 2000. https://doi.org/10.1161/01.CIR.101.23.e215

GUPTA, S. et al. Feature extraction using MFCC. Signal \& Image Processing : An International Journal (SIPIJ), v.4, n.4, 2013. https://doi.org/10.5121/sipij.2013.4408

HSU, C.-W.; CHANG, C.-C. \&. L. C.-J. A Practical Guide to Support Vector Classification. Taiwan: Department of Computer Science and Information Engineering, National Taiwan University., 2003.

KOLOURI, S.; PARK, S. R.; K. ROHDE, G. The Radon cumulative distribution transform and its application to image classification. CoRR, v. abs/1511.03206, 2015.

LEEB, R. et al. EEG-based "walking" of a tetraplegic in virtual reality. University College London, London - UK, 2004. https://doi.org/10.1155/2007/79642

LEEB, R. et al. Self-Paced (Asynchronous) BCl Control of a Wheelchair in Virtual Environments: A Case Study with a Tetraplegic. Computational Intelligence and Neuroscience, v. 2007 , p. 8,2007 . Article ID 79642.

LIN, C.-T. et al. EEG-Based Assessment of Driver Cognitive Responses in a Dynamic Virtual-Reality Driving Environment. IEEE Transactions on Biomedical Engineering, v. 54, n. 7, 2007 https://doi.org/10.1109/TBME.2007.891164
LINDASALWA, M.; BEGAM KASIM RAWTHAR, M.; L., E. Voice Recognition Algorithms using Mel Frequency Cepstral Coefficient (MFCC) and Dynamic Time Warping (DTW) Techniques. Journal of Computing, v.2, n. 3, 2010.

LOGAN, B. Mel frequency cepstral coefficients for music modeling. In: INTERNATIONAL SYMPOSIUM ON MUSIC INFORMATION RETRIEVAL (ISMIR) 2000. Massachusetts. Proceedings [...]. PLYMOUTH, MASSACHUSETTS, 2000. v. 28.

LUIZ A.; CARVALHO, J.; DIAS, D. Técnicas de Codificação de Voz Aplicadas em Sistemas Móveis Celulares. Department of Electrical Engineering School of Technology University of Brasilia, 2000.

MACHADO, V. B. Identificação Neural de Bloqueios Cardíacos. 1995 Dissertação (Mestrado). Universidade Federal do Espírito Santo Vitória, 1995.

MCCALLUM, A.; NIGAM, K. A comparison of event models for Naive Bayes text classification. AAAI-98 workshop on learning for text categorization. 752. [S.I.]: [s.n.], 1998.

PAL SINGH, P.; RANI, P. An Approach to Extract Feature using MFCC. IOSR Journal of Engineering (IOSRJEN), v. 4, n. 8, p. 21-25, $2014 . \quad$ https://doi.org/10.9790/3021$\underline{04812125}$

PARK, S. R. et al. The Cumulative Distribution Transform and Linear Pattern Classification. CoRR, 2015. abs/1507.05936.

R. FACHINI, A.; ROBERTO HEINEN, $M$. Aplicação de MFCC para modelar sons de instrumentos musicais. 1-4. 10.21528/CBIC2013-055. CONGRESSO BRASILEIRO DE INTELIGÊNCIA COMPUTACIONAL - CBIC. 11, 2013, 2016. https://doi.org/10.21528/CBIC2013-055 
RABINER, L.; JUANG, B.-H.; B, Y. Fundamentals of speech recognition. [S.I.]: Prentice-Hall Internatinal, 1993.

SCHALK, G. et al. BCI2000: A General-Purpose Brain-Computer Interface ( $\mathrm{BCl}$ ) System. IEEE Transactions on Biomedical Engineering $v$. 51, n. 6,p. 1034-1043, 2008, this paper received the Best Paper Award from IEEE TBME.], 2004. https://doi.org/10.1109/TBME.2004.827072

TEIXEIRA DOS SANTOS, $M$. et al. Processamento de sinais para 0 monitoramento do estado de ferramentas de corte usando redes neurais artificiais. In: SIMPÓSIO BRASILEIRO DE AUTOMAÇÃO INTELIGENTE - UFES, 1997, Vitória - ES. Anais [...]. Vitória, 1997.

TEIXEIRA DOS SANTOS, M.; ROSETTI DE ALMEIDA, A.; CHIEN CHING TU, C. Deteç̧ão do desgaste em fresas frontais utilizando redes neurais artificiais. In: CONGRESSO BRASILEIRO DE ENGENHARIA MECÂNICA, 15., 1999, Águas de Lindóia. Anais [...]. Águas de Lindóia, 1999.

TZANETAKIS, G.; COOK, P. Musical genre classification of audio signals. In: SPEECH AND AUDIO PROCESSING, IEEE TRANSACTIONS, 2002. p. 293-302. https://doi.org/10.1109/TSA.2002.800560

W., H. et al. An efficient mfcc extraction method in speech recognition. IN CIRCUITS AND SYSTEMS, 2006. ISCAS 2006. PROCEEDINGS. 2006 IEEE INTERNATIONAL SYMPOSIUM ON, PP. 4-PP, IEEE, 2006.

YEOM, S.-K.; SUK, H.-I.; LEE, S.-W. Person authentication from neural activity of facespecific visual self-representation. Pattern Recognition, v. 46, n. 4, p. 1159-1169, 2013. https://doi.org/10.1016/j.patcog.2012.10.02 $\underline{3}$

ZHAO, Q.; ZHANG, L.; CICHOCKI, A. EEG-based asynchronous $\mathrm{BCl}$ control of a car in $3 \mathrm{D}$ virtual reality environments. Chinese Science Bulletin, v.54, n. 1, p.78-87, 2009. https://doi.org/10.1007/s11434-008-0547-3

ZWICKER, E.; FASTL, H. Psychoacoustics: Facts and Models. [S.I.]: Springer, 1999. https://doi.org/10.1007/978-3-662-09562-1 